\title{
CALCUL DES ERREURS MOYENNES AFFECTANT \\ LES MESURES D'ÉTOILES DOUBLES VISUELLES
}

\author{
J. DOMMANGET \\ Observatoire Royal de Belgique, Bruxelles, Belgique
}

Au cours de recherches bibliographiques diverses, nous avons remarqué que bien des observateurs se sont servis et se servent encore de formules par trop approximatives pour calculer - lorsqu'ils le font - les erreurs moyennes affectant leurs mesures.

Nous croyons important et opportun de rappeler ici la formule correcte dont nous avons donné une démonstration à une autre occasion (1959).

Pour calculer l'erreur moyenne affectant la mesure d'une grandeur, il est nécessaire de considérer soit un grand nombre de mesures de cette grandeur, soit plusieurs groupes de petits nombres de mesures de grandeurs distinctes mais présentant rigoureusement les mêmes difficultés d'observation.

C'est suivant ce dernier schéma que l'on se voit forcé de procéder dans le cas des mesures d'étoiles doubles visuelles car seuls sont disponibles généralement, des groupes - en assez grand nombre - de quelques mesures seulement, relatifs à des binaires différentes.

Certains observateurs procèdent alors au calcul de l'erreur moyenne de poids un dans chaque groupe de mesures, puis, à la moyenne arithmétique simple ou pondérée de ces erreurs moyennes pour diverses classes de valeurs de $\theta$ et de $\varrho$, ou encore de $m$ ou de $\Delta m$, par exemple. Une meilleure façon de procéder consiste déjà à calculer la moyenne quadratique des erreurs moyennes de poids un des divers groupes, parce qu'elle correspond à l'erreur cherchée si le nombre de mesures individuelles est identique dans tous les groupes et que, dans le cas des mesures d'étoiles doubles visuelles, ce nombre - toujours petit - varie peu (ibidem, Formule 4).

Toutefois, si l'on désire tenir compte de la variation de ce nombre d'un groupe à l'autre, la formule correcte à utiliser est la même que celle considérée pour calculer l'erreur sur une équation de poids un, lors de la résolution d'un système d'équations linéaires par la méthode des moindres carrés. Elle s'écrit (ibidem, Formule 3)

$$
\varepsilon= \pm \sqrt{\frac{\sum_{n} p_{i} e_{i}^{\prime 2}}{n-\eta}},
$$

où $e_{i}^{\prime}$ est l'écart de la mesure d'indice $i$ à la moyenne du groupe auquel elle appartient, $p_{i}$, le poids accordé à cette mesure, $n$, le nombre total de mesures considérées et $\eta$, le nombre de groupes de mesures c'est-à-dire le nombre de moyennes.

Remarquons que dans presque tous les cas on peut poser: $p_{i}=1$.

\section{Bibliographie}

Dommanget, J.: 1959, 'Mesures micrométriques d'étoiles doubles visuelles effectuées de 1950 à 1954 à l'équatorial de $45 \mathrm{~cm}$ ', Ann. Obs. Roy. Belg. 3e Série 8, 89 . 